\title{
Terahertz optical elements for control of high-power laser irradiation
}

\author{
V.S. Pavelyev $^{1,2}$, A.N. Agafonov ${ }^{1,2}$, B.O. Volodkin ${ }^{1,2}$, K.N. Tukmakov ${ }^{1,2}$, B.A. Knyazev ${ }^{3,4}$, \\ Yu.Yu. Choporova ${ }^{3,4}$
}

${ }^{1}$ Samara University, Samara, Russia, nano@ssau.ru

${ }^{2}$ Image Processing Systems Institute, Russian Academy of Sciences, Samara, Russia

${ }^{3}$ Budker Institute of Nuclear Physics of SB RAS, Novosibirsk, Russia

${ }^{4}$ Novosibirsk State University, Novosibirsk, Russia

Appearance of the sources of coherent and high power $\mathrm{THz}$ radiation [1] opened new horizons for investigations in this frequency range [2]. High attention is focused on silicon diffractive optical elements (DOE), which are used for the beam manipulation [3-7]. The lithographic etching of a silicon substrate has been used in [3-7] to fabricate binary relief of diffractive optical elements. Binary silicon element [4] coated with the antireflection coating remained intact upon exposure to an average radiation power density of 4 $\mathrm{kW} / \mathrm{cm}^{2}$; the peak power in a 100 ps pulse was almost $8 \mathrm{MW} / \mathrm{cm}^{2}$. Experimental estimates of the diffraction efficiency of the elements coated with the antireflection coating [4] are in good agreement with theoretical estimates.

Such applications like imaging, material ablation, generation of continuous optical discharge, and even more exotic for the terahertz range application, namely the field ionization of individual atoms, require focusing of $\mathrm{THz}$ radiation [3-5], often with an enhanced focal depth [3].

Non-diffractive Bessel beams with angular orbital momentum (vortex beams) with different topological charges were formed by use of binary phase spiral axicons [6]. Binary phase axicon (BPA) with spiral zone structure and with aperture diameter of $100 \mathrm{~mm}$ (Fig. 1a,b) has been realized in [7] by technology similar to described in [3-6].

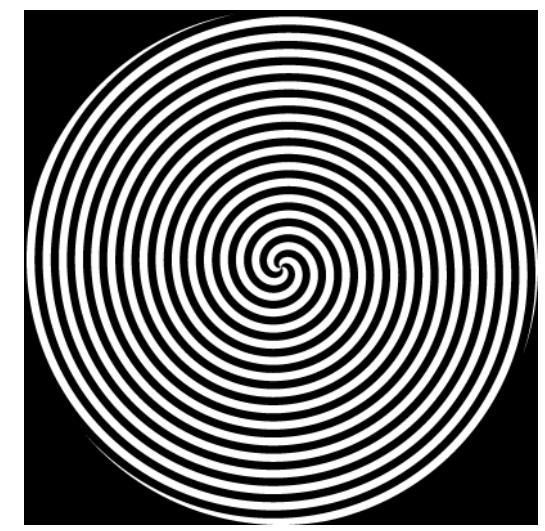

Fig. 1a. Calculated phase of binary diffractive optical element (black, 0; white, $\pi$ )

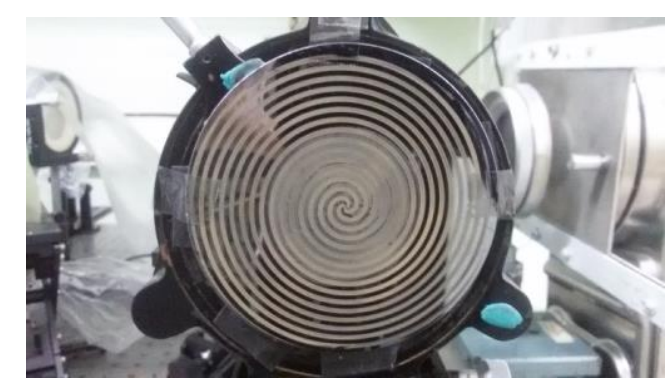

Fig. 1b. Photo of realized element

The research has been performed at workstations of the free electron laser NOVOFEL (Budker Institute of Nuclear Physics of SB RAS, Novosibirsk). A high power radiation (in a routine regime, the average power is $50-150 \mathrm{~W}$ ), a relatively narrow linewidth and the tunability of the radiation enable performing a wide variety of experiments. Vortex beam (Fig. 2) was formed from the NOVOFEL Gaussian beam (wavelength is of $129.5 \mu \mathrm{m}$ ) transformed by binary phase axicon (BPA) with spiral zone structures (Fig. 1) and with aperture diameter of $100 \mathrm{~mm}$.

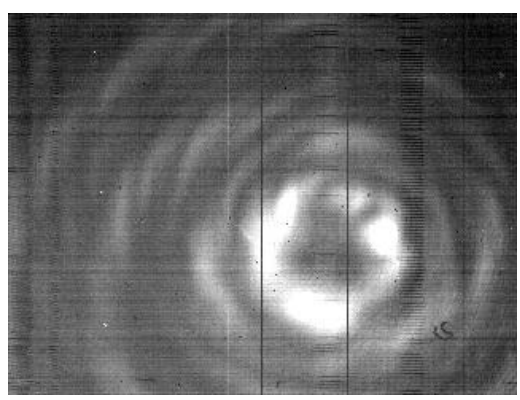

Fig. 2. Intensity distribution measured in the cross-section of beam formed by realized element (Fig. 1)

Vortex beams have great potential for use in free telecommunications and remote sensing [8].

However, lithographic etching has disadvantages in the case of multilevel elements, when an expensive and complicated procedure of alignment of photomask is required [8]. Binary (two-level) elements [37], in turn, have limited energy efficiency [8]. The laser ablation technology has been used in $[9,10]$ for fabrication of multilevel diffractive lens with high energy efficiency. However, diffractive optical elements are designed for working with monochromatic radiation of fixed wavelength [8] only. Fabrication of 
terahertz reflective free-form elements for transformation of high-power beams of Free Electron Laser has been considered in [11]. Aluminium elements (spherical and cylindrical mirrors (Fig. 3), reflective axicons) were fabricated by technology of micromilling [11].

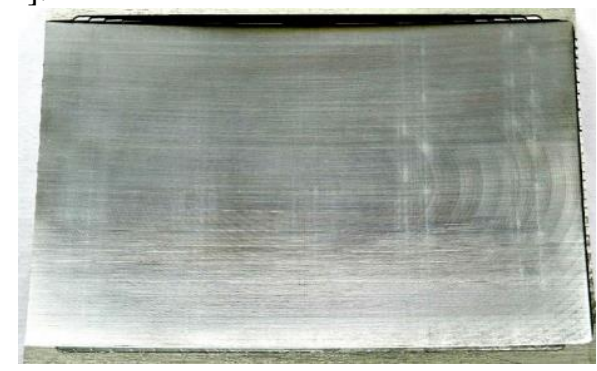

Fig. 3. Fabricated mirror

Realized optical elements were tested in the beam of NOVOFEL at the wavelength of $129.5 \mu \mathrm{m}$ (Fig.4). The measured diffractive efficiency of the spherical mirror (>94\%) is in good agreement with both numerical calculations and theoretical predictions.

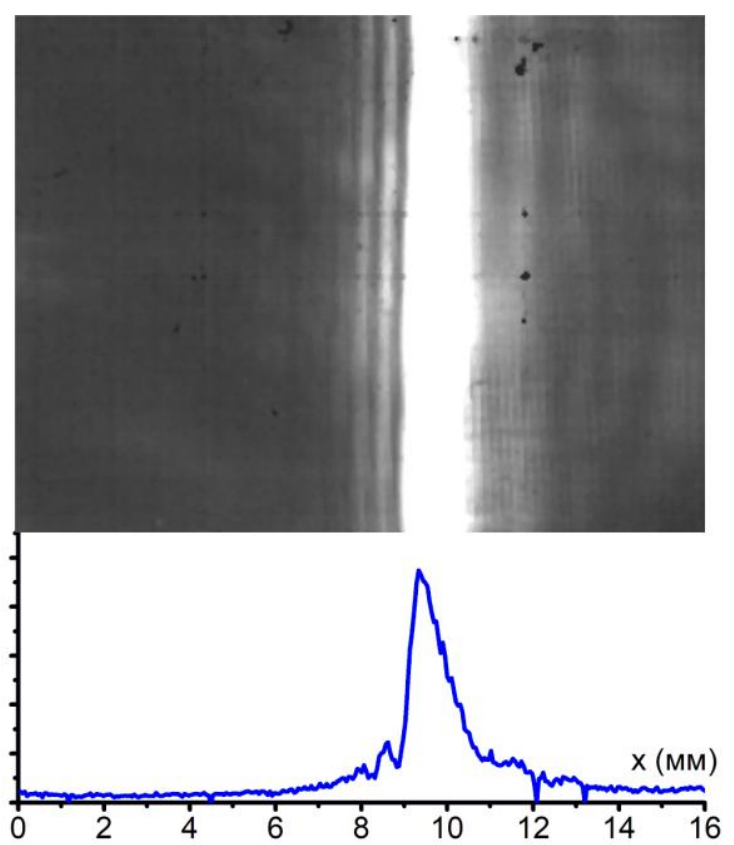

Fig. 4. Intensity distribution in the focal plane of cylindrical mirror

\section{Acknowledgements}

The elements were designed under support of the Ministry of Education and Science of the Russian Federation (project 16.7894.2017/6.7). The computer simulation of elements has been realized under support of RFBR grant 16-47-630560.

The terahertz beamline was designed under support of RSF grant 14-50-00080. The work was carried out in the collective research center supported by the Ministry of Education and Science of the Russian Federation, project RFMEFI62117X0012.

\section{References}

1. Knyazev, B.A., Kulipanov, G.N., Vinokurov, N.A. Novosibirsk terahertz free electron laser: instrumentation development and experimental achievements // Measurement Science and Technology. 2010. V. 21, No. 5. 054017, PP. 1-13 2. Kulipanov, G.N., Bagryanskaya, E.G., Chesnokov, E.N., Choporova, Yu.Yu., Gerasimov, V.V., Getmanov, Ya.V., Kiselev, S.L., Knyazev, B.A., Kubarev, V.V., Peltek, S.E., Popik, V.M., Salikova, T.V., Scheglov, M.A., Seredniakov, S.S., Shevchenko, O.A., Skrinsky, A.N., Veber, S.L., Vinokurov, N.A. Novosibirsk Free Electron Laser-Facility Description and Recent Experiments // IEEE Transactions on Terahertz Science and Technology. 2015. V. 5, No. 5, PP. 798-809

3. Agafonov, A.N., Volodkin, B.O., Kachalov, D.G., Knyazev, B.A., Kropotov, G.I., Tukmakov, K.N., Pavelyev, V.S., Tsypishka, D.I., Choporova, Yu.Yu., Kaveev, A.K. Focusing of Novosibirsk Free Electron Laser (NovoFEL) radiation into paraxial segment // Journal of Modern Optics. 2016. V. 63, No. 11, PP. 1051-1054.

4. Agafonov, A.N., Volodkin, B.O., Kaveev, A.K., Knyazev, B.A., Kropotov, G.I., Pavel'ev, V.S., Soifer, V.A., Tukmakov, K.N., Tsygankova, E.V., Choporova, Yu.Yu. Silicon diffractive optical elements for high-power monochromatic terahertz radiation // Optoelectronics, Instrumentation and Data Processing. 2013. V. 49, No. 2, PP. 189-195.

5. Agafonov, A.N., Volodkin, B.O., Volotovsky, S.G., Kaveev, A.K., Knyazev, B.A., Kropotov, G.I., Tykmakov, K.N., Pavelyev, V.S., Tsygankova, E.V., Tsypishka, D.I., Choporova, Yu.Yu. Silicon optics for focusing of terahertz laser radiation in a given two-dimensional domain// Computer Optics. 2013. V. 37, No. 4, PP. 464-470 (In Russ.).

6. Knyazev, B.A., ' Choporova, Yu.Yu., · Mitkov, M.S., . Pavelyev, V.S., · Volodkin, B.O. Generation of Terahertz Surface Plasmon Polaritons Using Nondiffractive Bessel Beams with Orbital Angular Momentum // Physical Review Letters. 2015. V. 115, N 16, art 163901.

7. Pavelyev, V.S., Volodkin, B.O., Tukmakov, K.N., Knyazev, B.A., Choporova, Yu.Yu. Transmissive Diffractive Microoptics for High-Power THz Laser Radiation // AIP Conference Proceedings. 2018. V. 1989, No. 1, art. 020025.

8. Computer Design of Diffractive Optics, edited by V.A. Soifer. Cambridge International Science \& Woodhead: 2012.

9. Volodkin, B.O., Knyazev, B.A., Kononenko, V.V., Kononenko, T.V., Konov, V.I., Pavelyev, V.S., Soifer, V.A., Tukmakov, K.N., Choporova, Yu.Yu. Fabrication of a multilevel $\mathrm{THz}$ Fresnel lens by femtosecond laser ablation // Quantum Electronics. 2015. V. 45, No. 10, PP. 933 -936. 10. Minkevičius, L., Indrišiūnas, S., Šniaukas, R., Voisiat, B., Janonis, V., Tamošiūnas, V., Kašalynas, I., Račiukaitis, G., Valušis, G. Terahertz multilevel phase Fresnel lenses fabricated by laser patterning of silicon // Optics Letters. 2017. V. 42, No. 10, PP. 1875-1878.

11. Pavelyev, V., Agafonov, A., Ahmetova, E., Platonov, V., Knyazev, B., Reflective free-form optical elements for focusing of high-power $\mathrm{THz}$ radiation // Book of abstracts of International Conference "Synchrotron and Free electron laser Radiation: generation and application" (SFR-2018), PP. 41-42. 